\title{
Modular FSO optical system design for classical and quantum optical communication systems
}

Shehryar Ishtiaq, Christopher Schmidt, Florian Moll

Shehryar Ishtiaq, Christopher Schmidt, Florian Moll, "Modular FSO optical system design for classical and quantum optical communication systems," Proc. SPIE 11852, International Conference on Space Optics — ICSO 2020, 118520G (11 June 2021); doi: 10.1117/12.2599161

SPIE Event: International Conference on Space Optics - ICSO 2021, 2021, Online Only 


\section{International Conference on Space Optics-ICSO 2020}

Virtual Conference

30 March-2 April 2021

Edited by Bruno Cugny, Zoran Sodnik, and Nikos Karafolas
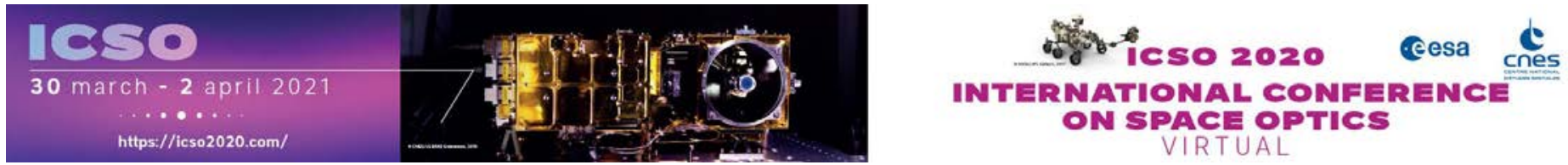

\section{Modular FSO optical system design for classical and quantum optical communication systems}

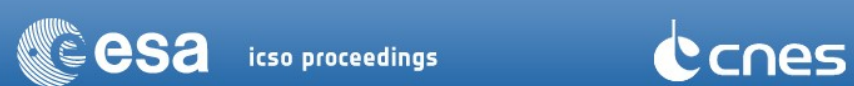




\title{
Modular FSO Optical System Design for Classical and Quantum Optical Communication Systems
}

\author{
Shehryar Ishtiaq, Christopher Schmidt, and Florian Moll \\ Optical Communication Group - Satellite Networks, Institute of Communication and \\ Navigation, German Aerospace Centre (DLR), Münchenerstr. 20, (82234) Weßling, Germany
}

\begin{abstract}
Optical communication systems are picking up pace in the various industries for its many scenarios in which it can be used. This includes satellite, aircraft and ground systems for which the DLR Institute of Communications and Navigation develops laser terminals. Free Space Optics (FSO) based optical systems have different applications ranging from terrestrial to airborne to space, and thus associated requirements to these application domains vary even though primary functional goals remain the same. Furthermore, quantum communication became of interest for its inherently secure key generation process that allows two nodes to securely communicate with each other and share secrets for long term security. Out of the different use cases of free-space optical laser terminal came the concept of having a modular FSO optical system design that could quickly adapt to the changing needs from mission to mission. The idea of a modularity appeals to many despite its challenges in the beginning, but in the long run the pros outweigh the cons. The concept was realized by carefully studying different requirements from various on-going and past projects and thereafter segmenting different aspects of the optical system based on three main categories, namely; the received path, the transmitted path and the shared path. To elaborate modularity in system; a Fast Steering Mirror (FSM) can be replaced with a fold mirror for a simpler design if it is not required, or an exchangeable CPA with integrated telescope mounted at the aperture of the optical bench to allow for different magnifications and ex-aperture beam diameter, all these without major impact on other subsystems. This greatly reduces the effort involved in re-evaluating system performance in the long run. In this study, the design and development of such an optical system is presented that lays its ground in the Institute of Communications and Navigation's OSIRIS project, but still continues to serve as the primary optical system design of choice for future projects.
\end{abstract}

Keywords: Laser Terminal, Optical Communication, Quantum Communication, System Design, Modular System

\section{INTRODUCTION}

The Institute of Communications and Navigation is working for more than 25 years in the field of free space optical communication. During this time, the program OSIRIS (Optical Space Infrared Downlink System) has been set up. ${ }^{1}$ The goal of this program is to develop compact and innovative optical communication payloads for small LEO satellites. While the first versions OSIRISv1 and OSIRISv2 on satellites Flying Laptop and BiROS respectively had a clear scientific goal, the ongoing developments target a series manufacturing together with an industrial partner. ${ }^{2-4}$

While OSIRISv1 and OSIRISv2 focused on a technology demonstration and proof of concept with data rates from $200 \mathrm{Mbps}$ up to $1 \mathrm{Gbps}$, the latest development in this row, OSIRISv3, is planned to reach data rates of 10 Gbps. ${ }^{5}$ OSIRISv3 will be demonstrated on Airbus' external payload platform Bartolomeo. ${ }^{6}$ OSIRISv3 has been developed as communication payload on small satellites for Direct To Earth (DTE) communication which is always sensitive to atmospheric effects. Therefore, to ensure successful data transmission, OSIRISv3 is equipped with an optical uplink channel to implement an Automated Repeat Request (ARQ) to retransmit lost data packets. ${ }^{7}$ For different satellite missions, different requirements have to be considered and consequently different configurations of the optical bench within the terminal. A modular system concept supports the idea

\footnotetext{
* Send correspondence to S.I., Shehryar.Ishtiaq@dlr.de; phone +49 (0)8153 28-3134; www.dlr.de/kn
} 
of adapting OSIRISv3 to different mission's needs. While the OSIRIS line of projects focused on classical optical communication, the Institute of Communications and Navigation in parallel also worked on quantum communication systems.

Quantum key distribution (QKD) is currently the most mature and therefore most interesting application in the field of quantum communications. Here quantum states are used to implement a protocol that enables two parties to securely agree on a common key. This key is often further used in known encryption techniques like AES or one-time pad. A ground based global-scale quantum network comes with its limitations and is near impractical as pointed out by Bedington et. al. ${ }^{8}$ Therefore, to realize the goal of a global quantum network for secure communication, satellite based QKD presents itself as a lucrative option. ${ }^{9}$

The physical layer is very similar in classical laser communications and quantum communications since the later usually uses photons for implementation of the quantum states. Therefore, the optical system of a laser terminal shares high synergies for both applications (classical laser communications and quantum communications). ${ }^{10}$ Within the project OSIRISv3, a laser terminal for LEO satellites is developed. Because of its high modularity, this concept forms the basis for a breadboard version of the laser terminal for demonstration of QKD secured data transmission in the framework of the project QuNET. In this paper we are presenting the design of such an optical system that realizes the synergies within the classical and quantum FSO communications, and uses the experience gained from past and existing projects in these domains to draw out similarities for design requirements.

\section{DESIGN METHODOLOGY}

The Institute of Communications and Navigation develops laser terminals for various platforms be it airborne, space or terrestrial application. It was realized from past projects, such as the one discussed by Nauerth et. al ${ }^{11}$ where air to ground QKD experiments were carried out as a close approximation of satellite to ground scenario, and on-going projects that the functional requirements are closely related and with the planned upcoming missions similar aspects could be expected. Out of this realization comes the idea of an FSO optical system where modularity and scalability sit as key design drivers in the development of the system. Though the initial effort would be large but in the long run the effort involved in designing custom optical systems for each of mentioned scenarios could be greatly reduced. With the modular FSO optical system discussed here we were able to tailor and apply to the needs of two independent projects namely; OSIRIS representing classical in-space optical communication application, and QuNET which is currently more leaned towards land-based quantum communication demonstration and experiments which perhaps in future take to airborne and space platforms as well.

The design methodology for the optical system stems from various on-going project needs and existing hardware of the institute. This also brings in the aspects and requirements coming from integration and compatibility to existing hardware and on-going CCSDS standard development. The optical system is to serve as a reference implementation of the CCSDS standard Optical On-Off-Keying (O3K). ${ }^{12}$ To ensure this compatibility and integration to existing hardware a few design requirements are discussed below. It is worth mentioning that while the system in basic configuration sees itself compliant to these aspects, it is also possible to scale up or down the system which is discussed in detail in section 5.1.

\subsection{Clear Aperture Selection}

For the initial sizing of optics existing hardware was carefully looked at and one key parameter i.e. clear aperture was found to be a defining and associative property. The clear aperture of an optic is used to defined to surface area in which the optical element would perform and deliver as specified optical quality. Since the optical system is to utilize commercially available optical elements as much as possible, this property was looked at as a ratio of mechanical diameter from several optics manufacturers and suppliers. After which a beam diameter of $15 \mathrm{~mm}$ was used in the designing of the optical system, which later went on to becoming the clear aperture diameter of the optical system itself.

Scalability aspects of the optical system meant that it would be possible to use the design in any size of optics as long as all elements were scaled by the same factor. However, to proceed with the design process and 
produce a prototype the size of optics would have to be defined which came from the dimensional constraints of the ongoing projects at the time. Individual optical elements used in the optical system however would go on to have larger clear apertures based on beam folds or their respective tilt and Field of View (FoV). This led to the selection of $25.4 \mathrm{~mm}$ optics in the system. For other elements which for most operational requirements would anyways be project and mission scenario based custom optics, such as focusing lenses, beam splitter and filters, this was not the case and a new norm had to be defined. In the case of the produced prototype after various opto-mechanical design considerations this was selected to be $20 \mathrm{~mm}$ mechanical diameter for focusing lenses and filters. For the beam splitter plates a dimension of $20 \mathrm{~mm} \times 26 \mathrm{~mm}$ with the substrate thickness being $3 \mathrm{~mm}$ was selected. Which in this size was found to be enough to achieve required optical quality over the clear aperture and suitable for most requirements on separation foreseen so far in the mentioned projects.

\subsection{Calibration and Alignment Concept}

For the alignment and calibration of the optical system it was important to be able to access all elements easily and if needed due to damaged coating or contamination easily replaceable and cleanable. Therefore, a significant negative space volume was to be left between the elements to allow for this to happen. However, after first design iterations this could be optimized to reduce overall optical system volume. This optimization took place in two steps which are based on ease of calibration and alignment stability.

Where for alignment stability the grouping of optical elements with dependent tolerances to single mechanical assembly took place. Which through mechanical tolerances ensured that the grouped optical elements don't displace with respect to each other and the use of adjustment pins along longest moment arms in opto-mechanical assembly ensured that the mounted assemblies hold alignment with respect to each other. These grouped assemblies also paved way for the reduction of overall volume and need for number of negative spaces. For ease of calibration it was ensured that in these grouped assemblies each optical element could be accessed and removed individually while being mounted to the optical bench. An example of this could be seen in figure 3(a) where the beam splitters mechanical assembly is shown which allows either of the beam splitters to be removed individually by removing the covering cap. Similar concept for the lens and filter assembly is shown in figure 3(b) where the cylindrical assembly in the center can be unscrewed to remove the pair of optical elements, or the retaining ring on either side of the cylindrical assembly can be unscrewed to remove individual elements. This mechanism also serves the purpose of calibrating the focusing lens to the sensor or receiver assembly by providing a compensator in the optical axis.

One desirable aspect in the optical system was also the ability to be able to perform self-calibration which would make sense for terminals which are in space or airborne where human intervention for calibration would not be accessible during operation. This requirement might be an overkill for the basic configuration and prototype testing, therefore it is added as a feature and discussed in detail in section 5.3.

\subsection{Optical Losses and Optimization}

It is safe to say that a lot is possible within a modular system including continuous optimization for reduction of optical losses, which were done from version to version of the optical system as projects changed. However, to have figures to start with a design approach and act as figures of merit for different trade-off studies in the development process, certain assumptions were made which are discussed here.

Since the optical system is being designed as a reference implementation of the CCSDS standards, we see the design tailor to the transmission wavelengths in the C-band and receiving wavelengths in the L-band. Particularly the selected design wavelength for transmission path was selected as $1550 \mathrm{~nm}$ and for the received path was selected as $1590 \mathrm{~nm}$.

Table 1 lists assumed values that were used for various optical elements for design development of the modular optical system concept. It is safe to say that various coatings on different substrates could yield different results but these values were found to be modest estimates based on market survey carried out at the time. 
Table 1: Assumed optical transmission losses and surface irregularities

\begin{tabular}{|c|c|}
\hline Optical Element & Assumed Transmission Loss \\
\hline Fold Mirrors & $98 \%$ \\
\hline Beam Splitters & $95 \%$ \\
\hline Filters & $95 \%$ \\
\hline Focusing Lens & $98 \%$ \\
\hline
\end{tabular}

\section{OPTICAL SYSTEM LAYOUT}

The optical system design can be divided up into three parts namely; transmission beam path, tracking beam path, and the received beam path. These are discussed in detail individually below. However, all the paths also share a common section which was designed together with the transmission path and hence main design driving requirements stem from there. This approach made sense as the transmission beam is typically going to have a Gaussian beam profile with requirements on divergences and beam width. Whereas, over long link distances the tracking and received beam as seen by an optical system typically has uniform beam profile in ideal case. The transmission beam was also realized as the one with strongest influence to the overall system performance as among the three discussed paths, deviations in beam profile and power of the transmission beam resulted in the most change in overall link quality. In the long run this approach made way for achieving the requirements on all paths in a balanced and systematic manner.

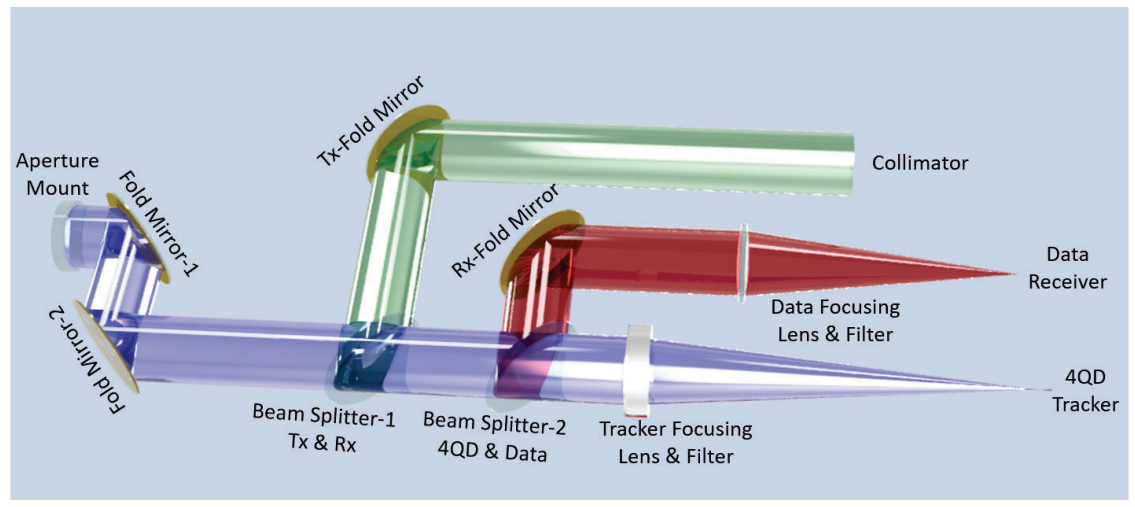

Figure 1: Optical System Layout Overview

\subsection{Transmission Beam Path}

In figure 2(a) the transmission beam path is highlighted and shown. If we follow the beam propagation, it can be seen that after leaving the fiber coupler the beam is reflected by a mirror assembly towards the first beam splitter assembly, referred to as $\mathrm{Tx} / \mathrm{Rx}$ beam splitter, responsible for the split between the transmission path and the other beams. After this the beam propagates through the common path which is comprised of two fold mirrors and passes through the exit aperture of the optical system. In section 4, we discuss the modularity in design where additional elements are added to meet various requirements; however, in its basic configuration the transmission path is design to have minimum optical elements to have as less losses as possible.

It is worth mentioning that the compensator foreseen in the transmission beam path is in the form of fine focus and tilt adjustment within the collimator assembly. In the case of OSIRIS project, the telescope lenses (mounted after the exit aperture of the optical bench) have an adjustment for divergence matching in the form of translation of the secondary lens in the optical axis. When needed this can be replaced by relay optics discussed in chapter 4 . In this basic configuration the transmission path has losses of approximately $10.5 \%$ for the designed wavelength and assumed losses from optical elements discussed earlier. 


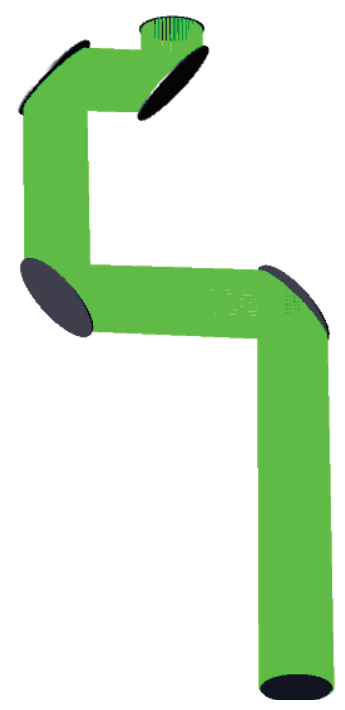

(a)

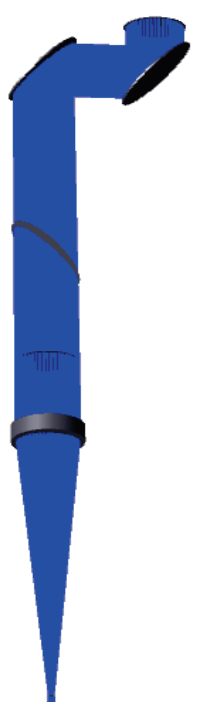

(b)

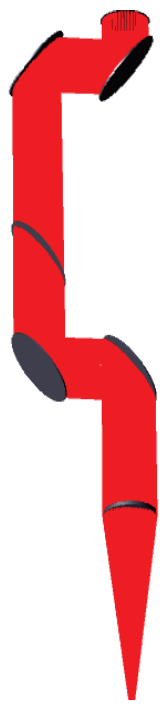

(c)

Figure 2: (a) Transmit Beam Path, (b) Tracking Beam Path, (c) Received Beam Path

\subsection{Tracking Beam Path}

After the shared path and splitting at $\mathrm{Tx} / \mathrm{Rx}$ beam splitter the tracking beam propagates further down the optical system as shown in figure 2(b). The beam is not folded and passes through the second beam splitter, referred to as Tracking/Data beam splitter, in order to keep the in-system angular deviations as less as possible. Folding the beam here would double the effect of any angular deviations which in turn covers more of sensor's Field of View (FoV) thus influencing the tracking performance. After this the beam propagates through a series of filter and focusing lens respectively housed within one assembly. The filter provides for essential noise cancellation and separation of any residual transmission or received back reflected beams. Whereas, the focusing lens focuses the beam on to the tracking sensor which in this optical system is a four-quadrant detector (4QD).

The assembly holding the filter and focusing lens is able to move in the optical axis direction allowing it to act a compensator in the tracking path. This would allow compensation for any changes in spot size diameter on the tracking sensor resulting from optical or mechanical manufacturing tolerances. This adjustment goes into the calibration process as a basic step to tune the spot size diameter on the tracking sensor ensuring a calibrated tracking path and as a corollary the tracking performance. The optical losses for the tracking path from entrance aperture till sensor under aforementioned assumptions is calculated as approximately $19.3 \%$ at design wavelength. However, for tracking performance it is also important to note aberrations in the system represented as a function of the wave-front error. The system must be diffraction limited and the spot shape must be checked together with the tracking sensor in simulation at design level that it does not induce unwanted behavior as the spot moves along the sensor plane.

\subsection{Received Beam Path}

At the Tracking/Data beam splitter the received beam is folded and separated from the tracking beam as shown in figure 2(c). This beam is then further folded down toward the filter and focusing lens assembly similar to the one mentioned in section 3.2 and plays similar role. However, the way the received beam path is different from the tracking path is that after the filter/focusing lens assembly there are variety of possibilities for data retrieval and otherwise.

In the case of OSIRIS project of DLR, an Avalanche Photodiode (APD) in combination with a short focal length lens was used. Whereas in the case of QuNET project this was replaced with a Commercial Off-The-Shelf (COTS) FSO data receiver mounted behind the optical bench in combination with a longer focal length lens. The path is also envisioned as follows; where the fold mirror replaced with a Fast Steering Mirror (FSM) could enable 
fiber coupling for future project needs including quantum experiments in a fiber-based system that is mounted external to the optical bench. This FSM positioning and modularity aspect is also highlighted in section 4 . The optical losses for the received path from entrance aperture until after focusing lens is calculated as approximately $21 \%$ under aforementioned assumptions for optical elements at design wavelength.

\section{MODULARITY IN DESIGN}

\subsection{Beam Splitting and Filtering}

The optical system design utilizes two beam splitters to achieve a well-defined separation between the different paths discussed in section 3. As for different project requirements different criterions would need to be adopted, in this topic we discuss the possibility of separating these paths under various criterions such as power, wavelength and polarization. In projects OSIRIS and QuNET, we have a clear distinction between the transmission beam (C-band) and the Received beam (L-band); therefore, a chromatic beam splitter was utilized. Even the difference between the tracking and received path could be realized in the same manner.

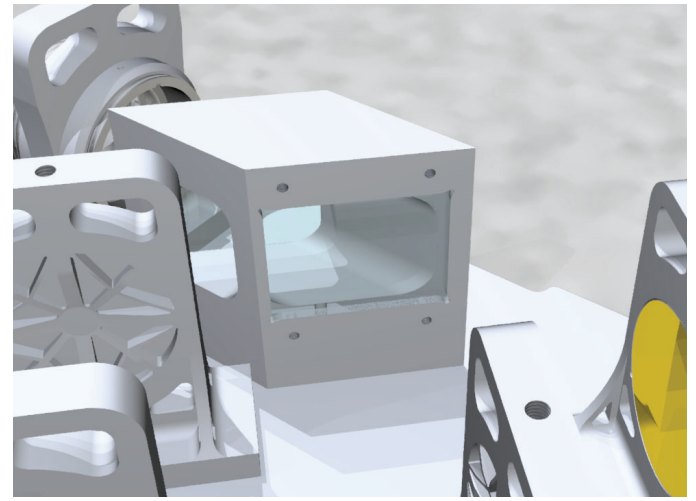

(a)

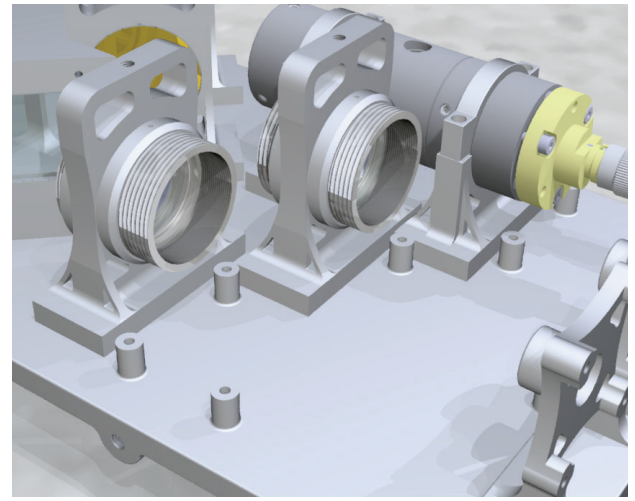

(b)

Figure 3: (a) Beam Splitter mounting Assembly, (b) Lens and Filter Mounting Assembly

However, the possibility for a $90-10$ power split was not fully ruled out. Due to the sensitivity of the 4QD receiver, it is possible to use the $90-10$ split for some mission profiles such as ground to LEO, where $10 \%$ of received power leading towards the $4 \mathrm{QD}$ receiver would still facilitate a stable tracking lock. It is also possible to switch these beam splitters in favor of a polarization dependent split should the requirements dictate, such as in the case of future quantum communication systems.

The two filters are situated ideally just before the focusing lenses, where the beam is still collimated, for the tracking and receiving paths in a combined lens and filter assembly. This allows for an easy integration and calibration with minimal mechanical assemblies utilized in the optical bench. For the transmission path, if required, it is possible to utilize the relay optics position holder discussed in section 4.4. Another filter place holder position that can be utilized and has influence over all beam paths is right at the opening aperture of the optical bench as shown in figure 4. This also acts as a good location to filter background light or as a place holder for waveplates which might be required in case of polarization optics.

\subsection{Collimator Sizing and Interchangeability}

The collimator mounting mechanism is designed to be easy to swap out existing collimator assembly and replace with another to achieve different transmission beam sizes and divergences. Between projects OSIRIS and QuNET the collimator assembly was exchanged to facilitate the different project requirements. A smaller beam size was required in project QuNET therefore the existing collimator was replaced with a smaller assembly held using the same c-shaped clamps as shown in figure 5 .

There is provision for longer collimator assemblies by utilizing two of such mounts, as was the case with OSIRIS project. This allows for a variety of beam sizes to be produced within the same optical bench which 


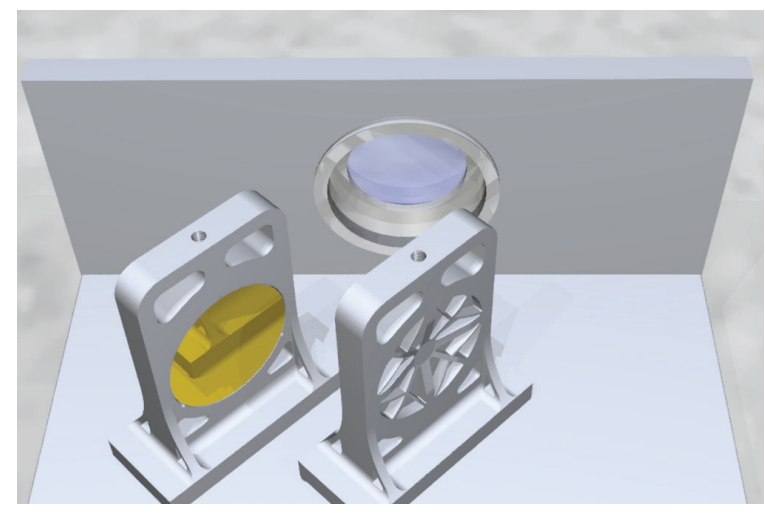

Figure 4: Aperture mount assembly including ring adaptor

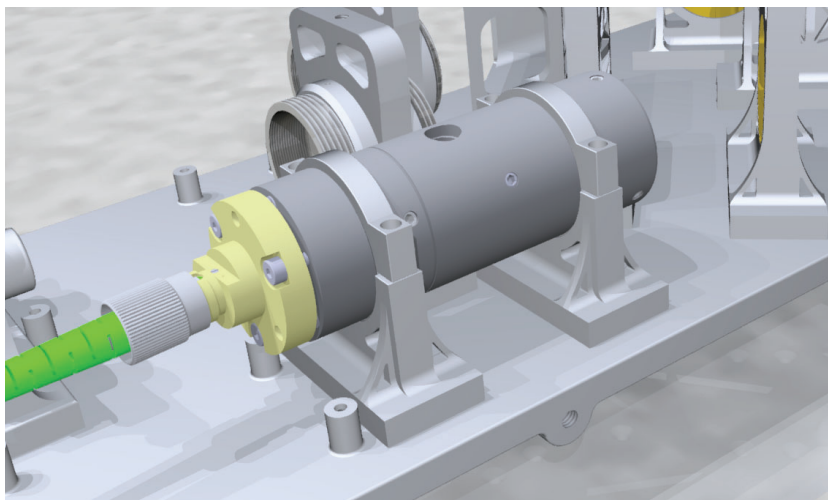

Figure 5: Collimator Mount Assembly

in turn reduces time and effort for prototyping and experimenting. However, keeping in mind the limitation of following optical elements and clear aperture size discussed in section 2.1. In case larger optics are required the scalability aspects of the optical bench come into play which are discussed in detail in section 5.1.

\subsection{Beam Steering}

The modularity of the optical system design also extends to future project needs as well such as inter-satellite links or similar scenarios where pointing stability requirements can be greater. There is only so much that a satellite body pointing or even coarse pointing assemblies can achieve. For those other scenarios FSMs serve as a good solution to fill the missing gaps in requirements. Therefore, the optical system design foresees the use of FSMs to do just that. In figure 6, the fold mirrors are marked to show as potential sites for such FSMs.

In figure 6(a), we see two fold mirrors either of which could be replaced with a FSM to meet pointing stability requirements mentioned earlier. The two sites serve to provide a certain degree of design freedom for the telescope as either of the locations can be used as a pupil reference point so that the later FSM is placed on the pupil of the telescope assembly. In OSIRIS we had a Galilean telescope with a magnification of $\mathrm{x} 2$ where the first site after entrance aperture was also possible to provide fine steering within the FoV of the sensor despite not having a pupil.

To account for relativistic effects resulting in angular deviation between the transmission and received beam over large link distances, figure 6(b) shows the site where the fold mirror after the collimator is replaced with such an FSM to provide a Point Ahead Angle (PAA).

In case a fiber coupling is required for fiber-based systems after the optical assembly, as foreseen in project QuNET, figure 6(c) indicates another position where the fold mirror could be replaced in favor of an FSM to achieve higher coupling efficiencies in combination with the feedback from the tracking sensor. 


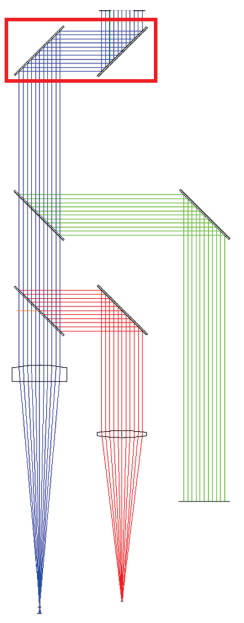

(a)

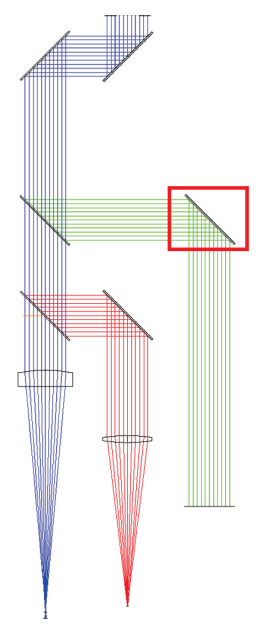

(b)

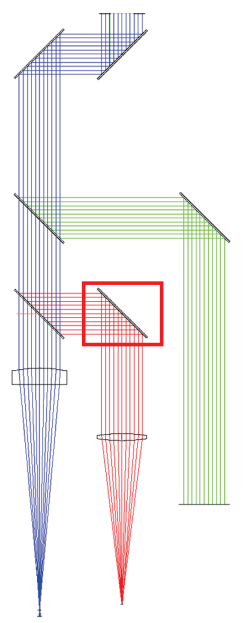

(c)

Figure 6: a) Fine Pointing Mirror sites, (b) Point Ahead Mirror site, (c) FSM for fiber coupling

\subsection{Relay Optics}

There are essentially two positions in the optical system design envisioned as short path relay optics place holders. One is as shown in figure 7(a) for the transmission path where it can be used for waveplates, mechanical apertures and beam shaping further after the beam leaves the collimator. This could also be helpful for in orbit calibration of transmit beam with low power optics on actuated assemblies to adjust the divergence and beam size of the transmit beam including utilisation of variable aperture for different beacon and data transmission beams. The other place holder for relay optics is one that influences all beam paths and is as shown in figure 7(b). This could serve all of the earlier mentioned purposes in addition to influencing all beam paths in the optical system.

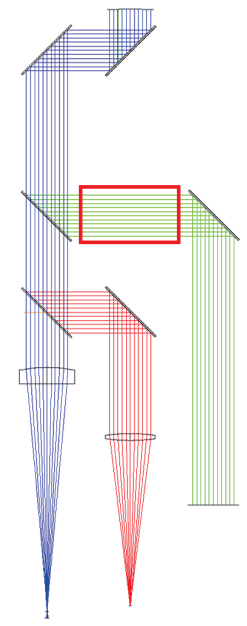

(a)

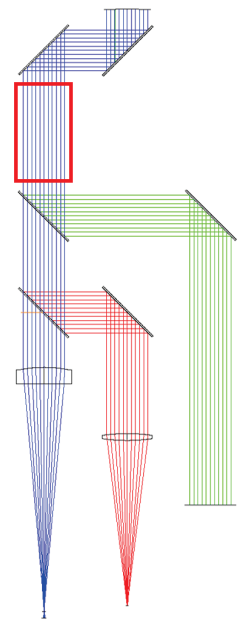

(b)

Figure 7: (a) Transmission path relay optics mounting position, (b) Relay optics mounting position for all paths 


\section{HARDWARE SPECIFICATION}

\subsection{Optical Bench Design}

The optical bench commonly is made from carbon fibre material due to its thermal performance as a result of low CTE and anisotropic properties. While the material and manufacturing process offered comparatively more design freedom, it was decided to utilize metal optical benches for the upcoming projects to facilitate the needs of rapid prototyping. This goes hand in hand with the modularity and scalability concept as one of the advantages the institute saw in this approach was to save development time by ease of scalability and preparedness of individual modules over a short period of time. Which was facilitated by the use of metal optical bench instead of the carbon fiber optical bench due to latter's complex manufacturing and relatively large lead times.

From space environment perspectives as well, the metal bench was favored due to challenging manufacturing control processes involved with a carbon fiber bench to ensure compatibility to outgassing requirements. Another aspect that favored metal optical bench was the thermal considerations of the ongoing project where it was identified that a good thermal coupling to the mechanical structure and the thermal control system would play a pivotal role in ensuring a good in-orbit performance.

Even within metals there are plenty of choices but keeping in mind the above points a trade-off study was performed between various materials under similar figures of merits. Aluminum 6061 came out as the preferred material of choice for the optical mounts and the bench itself however titanium will be used for some assemblies such as the lens and filter adaptors because of its close match of Coefficient of Thermal Expansion (CTE) to substrate materials used. This allowed us to match the CTE to the substrate and subsequently reduces stress build ups that could lead to catastrophic failures in case of the large thermal drifts expected in OSIRIS project.

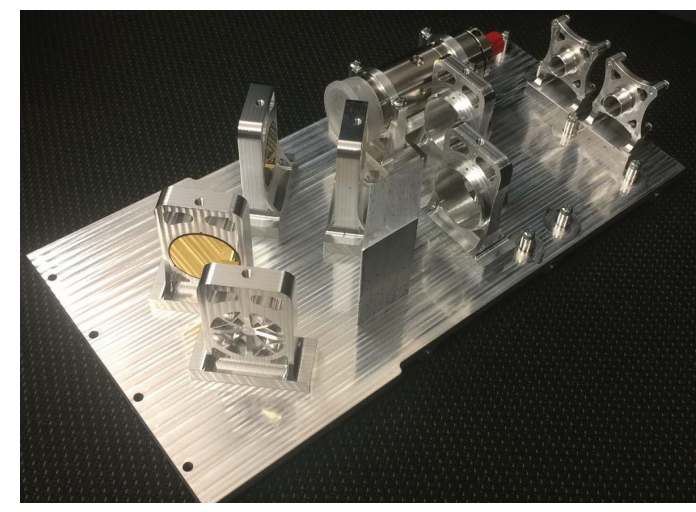

(a)

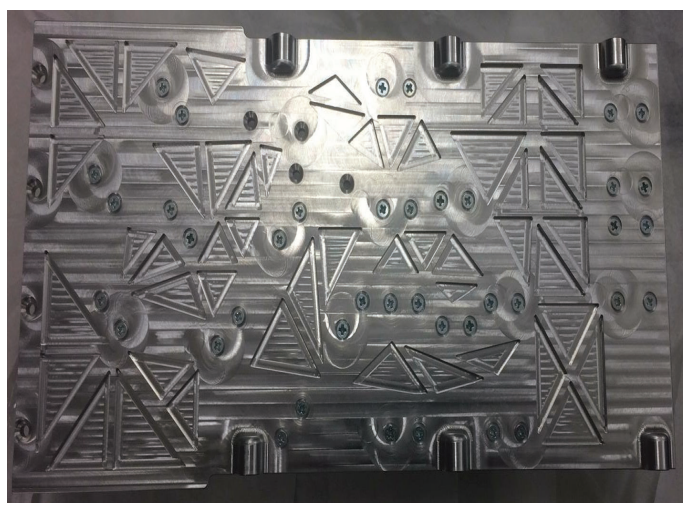

(b)

Figure 8: Modular Optical System Prototype (a) Top View, (b) Bottom View

Careful thought was given to the optimization of the optical mounts and the bench itself to route the heat flow optimally away from the optical elements and to ensure that the relative alignment between the optical elements is maintained. This resulted in the features that can be seen on the produced prototype as shown in figure 8. Although the image shows an open optical bench, there is a closing cover for contamination and venting control, which also acts as an electrical and optical fiber interface as discussed in section 5.2. Since in the current projects the optical bench is to be hard mounted to the mechanical assembly of the terminal, we could rely on the resulting thermally conductive path to be available and the mechanical system of the terminal itself acting as a heat sink.

\subsection{Electrical and Optical Interfaces}

Figure 9 shows two sites that can be used to place fiber connectors on the optical system covering's back side in order to connect to the collimator and coupler assemblies inside. This allows for an easy change of optical fibers and the optical system to be connected to multiple systems maintaining the goal of modularity even further. Besides the fiber connectors, the electrical harness feedthrough can also be seen which is used to connect to 
the electronics inside. This feedthrough, when not in use, can be closed in favor of using the 15 pin Micro-D connector also shown in the figure.

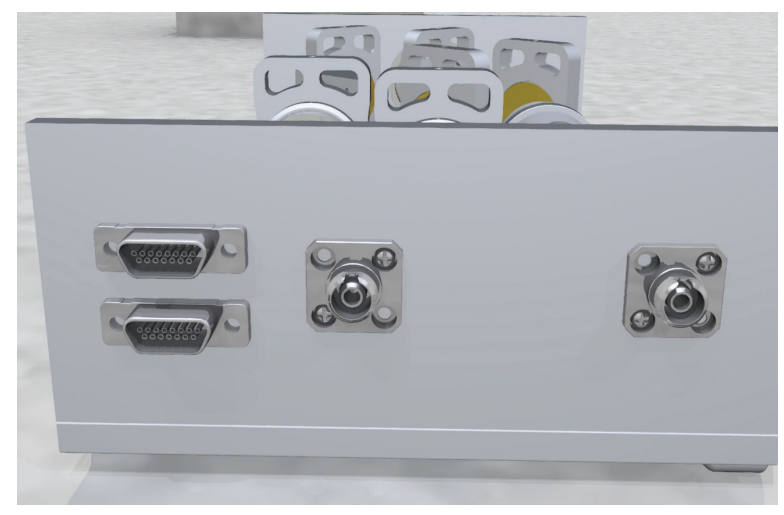

Figure 9: Electrical and optical interfaces on the optical system cover

In the OSIRIS project a central connecting assembly is used to connect different sub-systems together and for this a connector is placed to the side of the optical bench cover which uses an internal harness to provide easy removal of the cover. In the cover, there is an internal fiber routing milled out which is used to store and organize access fibers during integration. This way an easy and manageable integration concept could be realized which brings the optical system design to a very near plug and play system.

\subsection{Mitigation Strategies}

Several measures have been taken in the optical system design against not only background and stray light but also misalignments and self-calibration possibilities during operational scenarios which are highlighted here. The first measure of protection against unwanted light noise is that closed box design of the optical system itself, which provides contamination control as well. Though there are venting holes at the tail-end to provide a systematic venting of the contaminants inside in case of depressurization so that the optical elements are not in the path to collect them, they are also contoured and form a 3D routed path which is coated black to prevent any background or stray light from entering. In the figure 8 the optical system looks reflective. This image was taken before coating process as after the process the features are not so recognizable in an image.

The opening aperture of the optical system foresees a threaded insert for a combined baffle and filter assembly that can act as an additional control measure against background and stray light. Similarly, there are two baffles' in place between the focusing lens and the tracker or receiver assembly to prevent the stray light from reaching the sensors. Another aspect that helps to trap the back reflected light from optical surfaces is to mount the filters at a very slight angle. In our case, they are mounted a 2-degree tilt which is not enough to cause strong shifts in curve but enough to allow a shift of reflected beam that it gets trapped in the baffle or does not hit the sensor.

As it was mentioned earlier that the bench foresees a hard mounting to the mechanical system of the terminal around. While this approach works in most terrestrial and current scenario of the OSIRIS project, where we don't expect strong micro-vibrations from host and jitter requirements are relatively relaxed, in case of satellite or airborne terminals it is a possibility to have strong micro-vibration sources such as reaction wheels. In such scenarios there are two levels of measures in place; one is isolation on a terminal level which is not discussed in this paper, the other is isolation of the bench itself. For this the optical bench adopts to as shown in figure 10 to accommodate dampening mounts for further reduction of the micro-vibration frequencies after the terminal level measure. This also means that the bench would have to change its coating on the outside as the thermal coupling previously mentioned would not be available in a floating bench design and a combination of active and radiative cooling mechanisms would be needed.

Another mitigation measure put into place is the use of active relay optics for in-orbit calibration and performance optimization. In a self-calibration process the transmission path is used to send a beam that is 


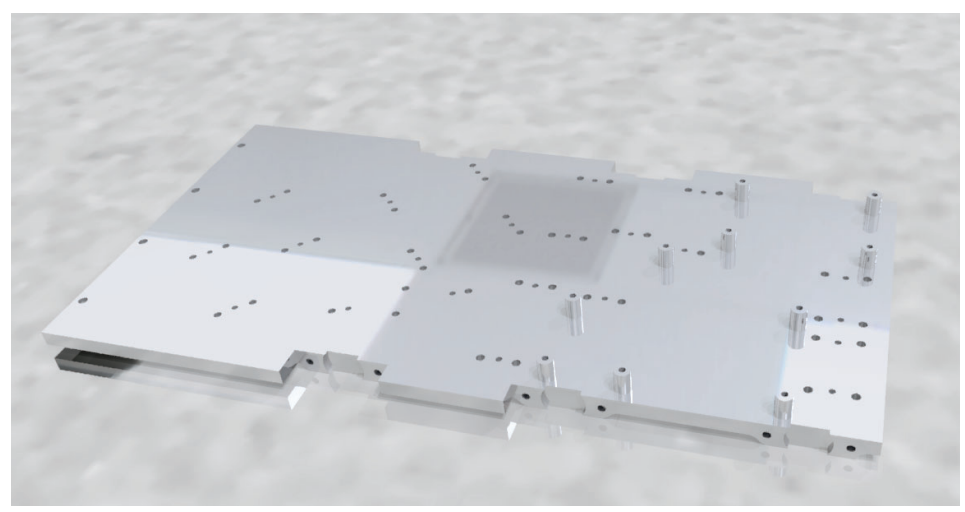

Figure 10: Optical Bench Modified for micro vibration dampener based mounting concept

not blocked by the tracking path filters and reflected back into the system by use of retro reflectors on the terminal, this of course means using the coarse pointing assembly in the terminal to point to the retro-reflector. With the reflected beam on the tracking sensor, the deviations can be calculated and corrected for by actuating the relay optics until performance is improved. Thereafter the relay optics in the transmission path could be re-adjusted to get the same output beam parameters for the transmission beam to ensure a good link. This in-orbit calibration concept could prove to be vital for assured mission success and continuous optimization to deliver peak performance till the mission's End Of Life (EOL).

\section{CONCLUSION \& FUTURE WORK}

The designed optical system with its scalability and modularity is able to serve as a good basis for the upcoming projects of the Institute of Communication and Navigation in classical and quantum communication. The amount of effort for optical designers in this way has been greatly reduced. Hence decreasing time for overall project development and achieving system maturity to required Technology Readiness Level (TRL) faster. While in the framework of current OSIRIS and QuNET projects the prototypes were developed in the systems basic configuration, further developments in the project also see utilization of the above-mentioned features in coming future. The modular optical system provides for opportunities to include a fine pointing assembly which has been brought to use and tested in initial phases of project QuNET.

Follow on missions also can look forward to including and testing of fiber coupling with the coupling efficiency optimization as a figure of merit. Also planned is the lab testing of the point ahead mirror in combination with the use of relay optics for in orbit calibration. Here, a deeper investigation into the concept is required where we would need to look at the aspects of relay optics design and achievable calibration adjustments using micro actuator-based system. Once the mentioned proof of concept demonstrations and testing have been performed, they could easily be incorporated in on going projects for an upgraded optical system. This approach of modular optical system allows for continuous system improvement with minimal consequences to the overall project hence could potentially be an appreciated change in the way we continue to develop laser communication terminals for future.

\section{ACKNOWLEDGEMENTS}

Authors would like to thank their colleagues of the Optical Communication Group at the Institute of Communications and Navigation - German Aerospace Centre (DLR) for their continued support through the various stages of development - from design to prototyping.

\section{REFERENCES}

[1] Fuchs, C., Schmidt, C., Keim, J., Moll, F., Rödiger, B., Lengowski, M., Gaißer, S., and Giggenbach, D., "Update on DLR's OSIRIS program and first results of OSIRISv1 on flying laptop," in [Free-Space Laser Communications XXXI], 10910, 109100S, International Society for Optics and Photonics (2019). 
[2] Fuchs, C., Moll, F., Giggenbach, D., Schmidt, C., Keim, J., and Gaisser, S., "OSIRISv1 on flying laptop: Measurement results and outlook," in [2019 IEEE International Conference on Space Optical Systems and Applications (ICSOS)], 1-5, IEEE (2019).

[3] Schmidt, C., Brechtelsbauer, M., Rein, F., and Fuchs, C., "OSIRIS payload for DLR's BiROS satellite," in [International Conference on Space Optical Systems and Applications 2014], (2014).

[4] Gaisser, S., Mohr, U., Keim, J., Triloff, D., and Klinkner, S., "Improvements in attitude determination and control of the small satellite flying laptop," in [33 ${ }^{\text {rd }}$ Annual AIAA/USUConference on Small Satellites], (2019).

[5] "First high capacity space-to-ground laser communications system for the european external ISS platform," Geospatial World (2018-03-26).

[6] Steimle, C., Walz, C., Fuchs, C., Pederson, D., and Lombardi, C., "Bartolomeo external platform entering into commercial service," in [International Astronautical Congress (IAC)], (2019).

[7] Knopp, M. T., Spoerl, A., Gnat, M., Rossmanith, G., Huber, F., Fuchs, C., and Giggenbach, D., "Towards the utilization of optical ground-to-space links for low earth orbiting spacecraft," Acta Astronautica 166, 147-155 (2020).

[8] Bedington, R., Arrazola, J. M., and Ling, A., "Progress in satellite quantum key distribution," npj Quantum Information 3(1), 1-13 (2017).

[9] Liao, S. K., Cai, W. Q., Liu, W. Y., Zhang, L., Li, Y., Ren, J. G., Yin, J., Shen, Q., Cao, Y., Li, Z. P., et al., "Satellite-to-ground quantum key distribution," Nature 549(7670), 43-47 (2017).

[10] Moll, F., Weinfurter, H., Rau, M., Schmidt, C., Melén, G., Vogl, T., Nauerth, S., and Fuchs, C., "Aerospace laser communications technology as enabler for worldwide quantum key distribution," in [Quantum Optics], 9900, 99000K, International Society for Optics and Photonics (2016).

[11] Nauerth, S., Moll, F., Rau, M., Fuchs, C., Horwath, J., Frick, S., and Weinfurter, H., "Air-to-ground quantum communication," Nature Photonics 7(5), 382-386 (2013).

[12] CCSDS 141.0-B-1, "Optical communications physical layer," Recommendation Standard, Consultative Committee for Space Data Systems (2019). 